\title{
Fiber-optic flow sensors for high-temperature environment operation up to $800^{\circ} \mathrm{C}$
}

\author{
Rongzhang Chen, Aidong Yan, Qingqing Wang, and Kevin P. Chen* \\ Department of Electrical and Computer Engineering, University of Pittsburgh, Pittsburgh, Pennsylvania 15213, USA \\ *Corresponding author: kchen@engr.pitt.edu
}

Received March 28, 2014; revised April 24, 2014; accepted May 6, 2014;

posted May 7, 2014 (Doc. ID 209083); published June 27, 2014

\begin{abstract}
This Letter presents an all-optical high-temperature flow sensor based on hot-wire anemometry. High-attenuation fibers (HAFs) were used as the heating elements. High-temperature-stable regenerated fiber Bragg gratings were inscribed in HAFs and in standard telecom fibers as temperature sensors. Using in-fiber light as both the heating power source and the interrogation light source, regenerative fiber Bragg grating sensors were used to gauge the heat transfer from an optically powered heating element induced by the gas flow. Reliable gas flow measurements were demonstrated between $0.066 \mathrm{~m} / \mathrm{s}$ and $0.66 \mathrm{~m} / \mathrm{s}$ from the room temperature to $800^{\circ} \mathrm{C}$. This Letter presents a compact, low-cost, and multiflexible approach to measure gas flow for high-temperature harsh environments. (C) 2014 Optical Society of America

OCIS codes: (060.3735) Fiber Bragg gratings; (060.2370) Fiber optics sensors; (280.2490) Flow diagnostics.

http://dx.doi.org/10.1364/OL.39.003966
\end{abstract}

Gas flow measurement plays important roles in various industrial sectors. It provides vital information for a large number of applications such as process controls, fossil fuel and nuclear electric power generation, transportation, and environment monitoring. To perform flow measurements, a large number of flow sensors based on various mechanical, electronic, and microelectromechanical system (MEMS) structures [1] have been developed. These sensors can perform effective flow measurements at room temperature or slightly elevated temperatures (e.g., $<200^{\circ} \mathrm{C}$ ). However, a number of industrial and aerospace applications demand flow sensors with much higher operational temperatures $\left(>500^{\circ} \mathrm{C}\right)$. These are not attainable by current state-of-the-art technology, such as MEMS. To address this technical challenge, this Letter presents a low-cost and compact all-optical-fiber flow sensing technique that can provide rapid and accurate gas flow measurements from room temperature to $800^{\circ} \mathrm{C}$. This is, to our best knowledge, the highest operational temperature for a flow sensor.

Fiber-optic sensors are well-known for their resilience in many harsh conditions including in high-temperature, corrosive, and strong electromagnetic environments. Over the last decade, various optical-fiber-based flow sensors have been reported, largely based on two schemes: fiber optical interferometry $[2,3]$ and optical hot-wire anemometry (optical HWA). Among different designs of optical flow sensors, the HWA is one of the most widely adopted flow measurement techniques, thanks to its simplicity and reliability. HWA-based flow sensors determine flow rate by measuring heat transfer between a heated element and adjacent temperature sensors [4].

Compared with electrically heated HWA flow sensors, fiber-optic sensors can also be heated optically to perform flow measurements. Using various optical coupling schemes [ $\underline{5}-11]$, in-fiber light has been used to heat up a section of fiber containing a sensing element (e.g., a fiber Bragg grating or FBG) to perform temperature measurements. The optical HWA can be more resilient than those using the electric heating for high-temperature applications. This is because the optical fiber, as a power delivery cable, can sustain much higher temperatures than metal wires.

In this Letter, we demonstrate an all-fiber hightemperature flow sensor using the optical HWA rated for $800^{\circ} \mathrm{C}$ operation. The sensor consists of an in-fiber optical heating element using high-attenuation fibers (HAFs). High-temperature-stable regenerated FBGs (RFBG) [12-14] were induced in HAFs and standard telecom fibers (SMF-28) as temperature sensors to gauge flow-induced heat transfers. The schematic of the experiment setup is shown in Fig. 1. A high power erbiumdoped fiber amplifier (EDFA) was built to provide up to $700-\mathrm{mW}$ optical power to heat the HAF via a circulator. A RFBG in HAF was used to measure the temperature of the optical heating elements. Another RFBG formed in SMF-28 located at the upper stream of the gas flow was used to measure the ambient temperature surrounding the heating element.

The wavelengths of FBG sensors were monitored by an optical spectrum analyzer (OSA, Agilent 86140B with $70 \mathrm{pm}$ resolution). A tube furnace was used to provide high-temperature environments for sensor testing. During measurements, the fiber sensor was inserted in a quartz tube (OD, $6 \mathrm{~mm}$, ID, $4 \mathrm{~mm}$ ). At the input side, $\mathrm{N}_{2}$ gas was introduced through a tee tube fitting. The fiber was sealed using a rubber ferrule. At the exit of the flow tube, the fiber was placed on a tapered v-groove fiber holder (Thorlab HFV002) to ensure that no contact

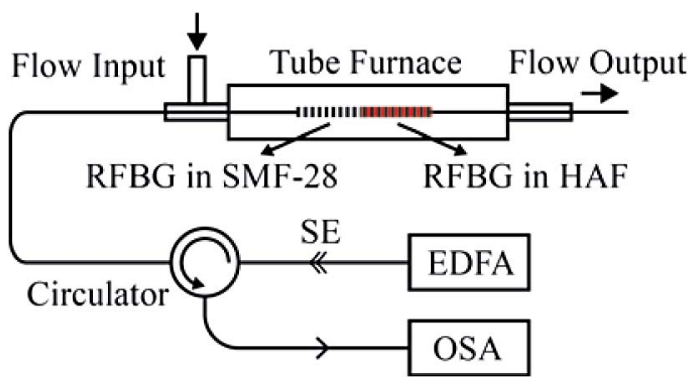

Fig. 1. Schematic of the experiment setup for sensor calibration and characterization. 
between the fiber and inner wall of the tube was made. A calibrated mass flow controller regulated the input flow rate of $\mathrm{N}_{2}$ gas. The sensor was tested under flow rates from 50 cubic centimeters (CCM) to $500 \mathrm{CCM}$, corresponding to mean flow velocity at the entry of the quartz tube ranging from $0.066 \mathrm{~m} / \mathrm{s}$ to $0.66 \mathrm{~m} / \mathrm{s}$. At this velocity range, the gas flow inside the quartz tube is considered as laminar only [ $\underline{15}]$.

Unlike conventional Type I FBGs which normally degrade rapidly at temperatures, RFBGs can be stable at the same temperature where it is regenerated $\left(800^{\circ} \mathrm{C}-\right.$ $1295^{\circ} \mathrm{C}$ [14]). This technique provides a low-cost approach to fabricate various sensors capable of hightemperature operations. To fabricate the fiber sensor based on regenerated gratings, two $L=1 \mathrm{~cm}$ long seed FBGs with $\lambda_{\text {Bragg }} \sim 1543.55 \mathrm{~nm}$ and $\lambda_{\text {Bragg }} \sim 1546.75 \mathrm{~nm}$ were inscribed in SMF-28 and HAF (CoreActive, attenuation coefficient $1 \mathrm{~dB} / \mathrm{cm}$ ) respectively. To enhance their photosensitivity, both optical fibers were soaked in a hydrogen chamber at $T \sim 25^{\circ} \mathrm{C}, P \sim 2400$ psi $(165$ bars) for about 1 week before the laser inscription. A $248 \mathrm{~nm} \mathrm{KrF}$ excimer laser with pulse fluence $f_{\text {pulse }} \sim$ $50 \mathrm{~mJ} / \mathrm{cm}^{2}$ and repetition rate $R R=20 \mathrm{~Hz}$ was used to inscribe the FBGs. A cumulative fluence of $f_{\text {cum }}=$ $0.09 \mathrm{~kJ} / \mathrm{cm}^{2}$ was assigned to produce the FBG in SMF28 while $f_{\text {cum }}=1.2 \mathrm{~kJ} / \mathrm{cm}^{2}$ was applied to HAF. These actions led to the FBG spectra shown in the left inset of Fig. 2. After the inscription, the samples were annealed at $120^{\circ} \mathrm{C}$ for $t \sim 12 \mathrm{~h}$. These two FBGs were then fusion spliced together to form the optical flow sensor as demonstrated in Fig. 1 .

The regeneration process starts from a posthydrogenatioon process [16]. Fiber sensor samples were first put back into the hydrogen chamber for posthydrogen-loading at 2400 psi to enhance its photosensitivity. This is a key process for successful regeneration, especially for FBGs in HAFs. The post-hydrogen-loading lasted for $t=1$ week. Then the fiber sample was placed in a box furnace for the thermal regeneration. A typical

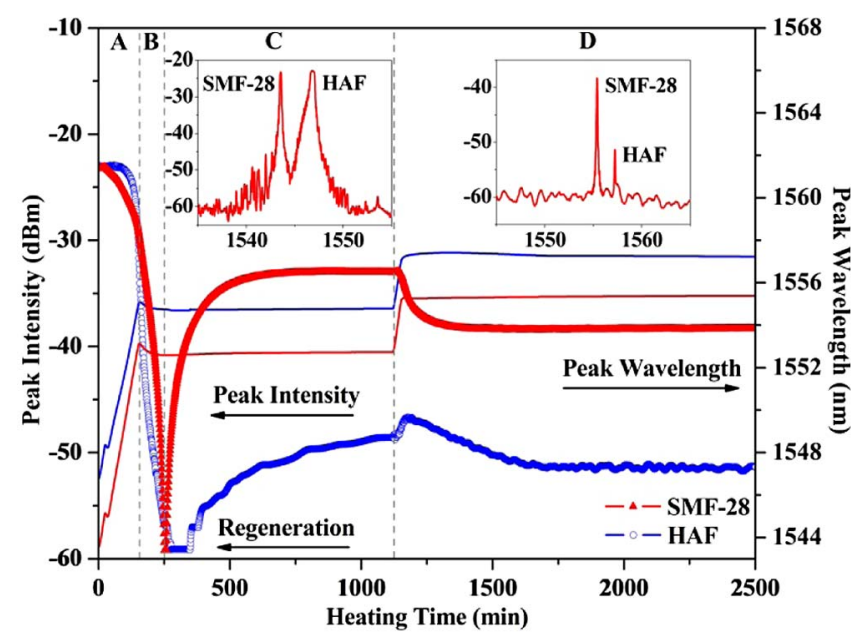

Fig. 2. Changes of grating strengths and resonant wavelengths during the regeneration process for FBG in SMF-28 (red) and $\mathrm{HAF}$ (blue) respectively. The dot traces are for FBG peak intensity while line traces are for FBG wavelength. Insets, reflection spectra of the seeds at room temperature $\left(T=25^{\circ} \mathrm{C}\right)$ and regenerated gratings at $1000^{\circ} \mathrm{C}$. regeneration process is shown in Fig. 2 . The temperature of the furnace was isochronally increased to $850^{\circ} \mathrm{C}$ with a rate of $5^{\circ} \mathrm{C} / \mathrm{min}$ in step $\mathrm{A}$ and then held steady in both steps $\mathrm{B}$ and $\mathrm{C}$ for isothermal annealing. FBGs in both SMF-28 and HAF were annealed rapidly as the temperature rose (step A), however, with different decay patterns. This is probably due to double-cladding fiber structures in HAF. During the grating erasure, the onset of a blue shift in $\lambda_{\text {Bragg }}$ is attributed to the thermal relaxation of UV-induced average refraction index change $\Delta n_{\mathrm{DC}}$. The full erasure of both FBGs occurred at the end of step $\mathrm{B}$, which lasted $t \sim 250 \mathrm{~min}$. This was followed by their subsequent grating regeneration in step C. Figure 2 shows that the FBG in SMF-28 was regenerated much faster than that in HAF. When both RFBGs approached their steady states after $t \sim 870 \mathrm{~min}$ of the thermal annealing at $T=850^{\circ} \mathrm{C}$, the furnace temperature was elevated to $1000^{\circ} \mathrm{C}$ for RFBG stabilization with the same rate of $5^{\circ} \mathrm{C} / \mathrm{min}$ as shown in step D. Both FBGs were finally stabilized after $t \sim 580 \mathrm{~min}$ of isothermal annealing at $1000^{\circ} \mathrm{C}$.

Once FBGs were regenerated, the flow sensor was characterized using the experiment setup shown in Fig. 1. The thermal responses of the two gratings were first measured and presented in Fig. 3. RFBGs in both HAF and SMF-28 exhibit slightly different thermo-optic coefficients for temperature from room temperature to $300^{\circ} \mathrm{C}$ and from $300^{\circ} \mathrm{C}$ to $800^{\circ} \mathrm{C}$ [17] where piecewise linear regression was used to evaluate the wavelengthtemperature coefficients $d \lambda / d T$ of FBGs in two temperature ranges as shown in Fig. 3 . The wavelengthtemperature coefficients determined in Fig. 3 are important to gauge optical heating performance of $\overline{\mathrm{H}} \mathrm{AFs}$.

The optical heating of the HAF sensor under different ambient temperatures was quantified by injecting optical power from the EDFA from 0 to $700 \mathrm{~mW}$. Not all optical power was absorbed by the HAF. It was estimated that the optical power absorbed by the 1-cm HAF was $14 \mathrm{~mW} / \mathrm{mm}$ with 700-mW input power. The optical heating performance of the HAF from the room temperature to $800^{\circ} \mathrm{C}$ is presented in Fig. 4 . The largest shift of resonant wavelength for the RFBBG in HAF is equal to

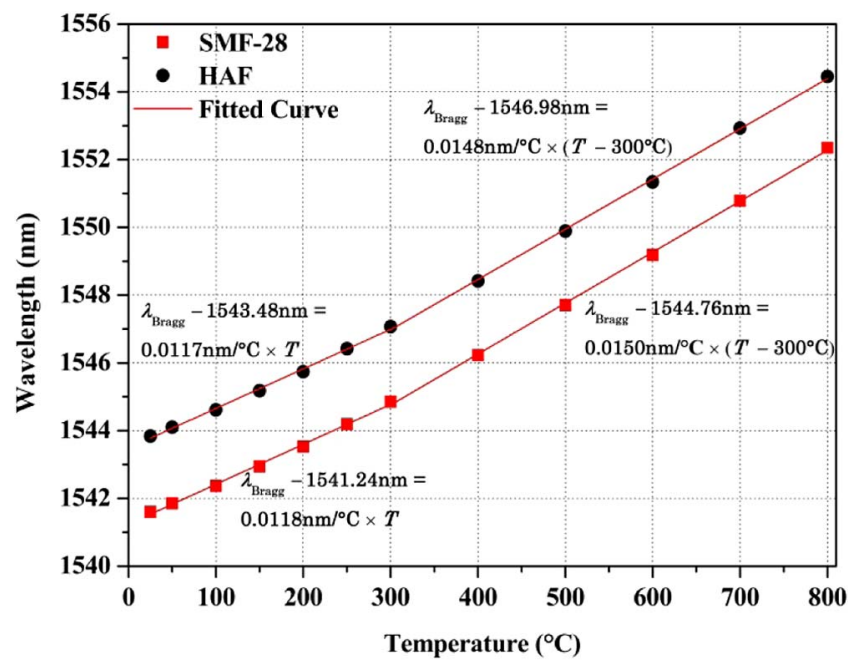

Fig. 3. Resonant wavelength versus ambient temperature for both RFBGs. 


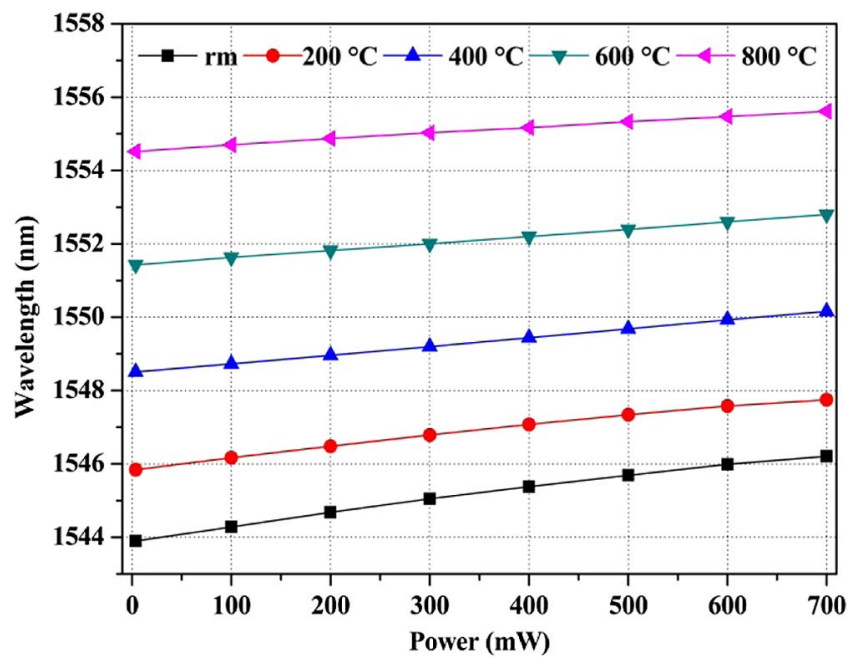

Fig. 4. Heating performance of the HAF under different ambient temperatures.

$\Delta \lambda=2.31 \mathrm{~nm}$, corresponding to $\Delta T=197.44^{\circ} \mathrm{C}$ at the room temperature. As ambient temperature increases, the optical heating becomes less efficient. At $800^{\circ} \mathrm{C}$ the shift of resonant wavelength was reduced to $\Delta \lambda=$ $1.09 \mathrm{~nm}$ using $700-\mathrm{mW}$ optical power, corresponding to $\Delta T=73.65^{\circ} \mathrm{C}$.

The operations of the flow sensor at the room temperature and at $800^{\circ} \mathrm{C}$ are shown in Fig. $\underline{5}$. Reflection spectra for RFBGs in SMF-28 and HAF in ambient temperatures are shown in black traces. When the HAF section of the fiber absorbed optical power of $14 \mathrm{~mW} / \mathrm{mm}$ at room temperature [Fig. 5(a)], the RFBG wavelength in the HAF shifted for $\Delta \lambda_{3}=2.31 \mathrm{~nm}\left(\Delta T=197.44^{\circ} \mathrm{C}\right)$. The upper stream RFBG in SMF-28 also experienced a small red shift in resonant wavelength for $\Delta \lambda_{1}=0.13 \mathrm{~nm}$, equivalent to temperature rise $\Delta T \sim 11^{\circ} \mathrm{C}$ as shown in Fig. 5(a) that was largely due to the thermal conduction between two grating areas. The temperature of this upper stream RFBG sensor would be used as the reference to gauge the ambient temperature surrounding the heated element (HAF). Thereafter, the flow of $\mathrm{N}_{2}$ was applied with a rate of $500 \mathrm{CCM}(0.66 \mathrm{~m} / \mathrm{s})$, where convection cooling becomes the major factor responsible for heat loss of the HAF. The resonant wavelength of the upper stream RFBG in SMF-28 shifted back toward its original by $\Delta \lambda_{2}=-0.15 \mathrm{~nm}$, lowering the surface temperature of the sensor to $T=23.31^{\circ} \mathrm{C}$ which was nearly $2^{\circ} \mathrm{C}$ lower than the preset ambient temperature $T=25^{\circ} \mathrm{C}$, probably due to the temperature drop of decompressed $\mathrm{N}_{2}$ itself. Meanwhile, the RFBG of HAF incurred a great blue shift in resonant wavelength for $\Delta \lambda_{4}=-0.71 \mathrm{~nm}$, corresponding to a temperature drop for $\Delta T=60.68^{\circ} \mathrm{C}$.

At $T=800^{\circ} \mathrm{C}$, the absorption of $14 \mathrm{~mW} / \mathrm{mm}$ optical power produced smaller wavelength shifts for both the reference RFBG $\left(\Delta \lambda_{1}=0.06 \mathrm{~nm}\right)$ and the RFBG in HAF $\left(\Delta \lambda_{3}=1.09 \mathrm{~nm}\right)$ as shown in Fig. 5(b). The introduction of 500-CCM $\mathrm{N}_{2}$ gas flow shifted the wavelength of the reference RFBG back to its original position $\left(\Delta \lambda_{2}=-0.06 \mathrm{~nm}\right)$. A blue shift $\Delta \lambda_{4}=-0.15 \mathrm{~nm}$ of the RFBG wavelength in HAF was observed, corresponding to a temperature drop $\Delta T=-10.14^{\circ} \mathrm{C}$ of the optically heated HAF. The temperature drops measured by the
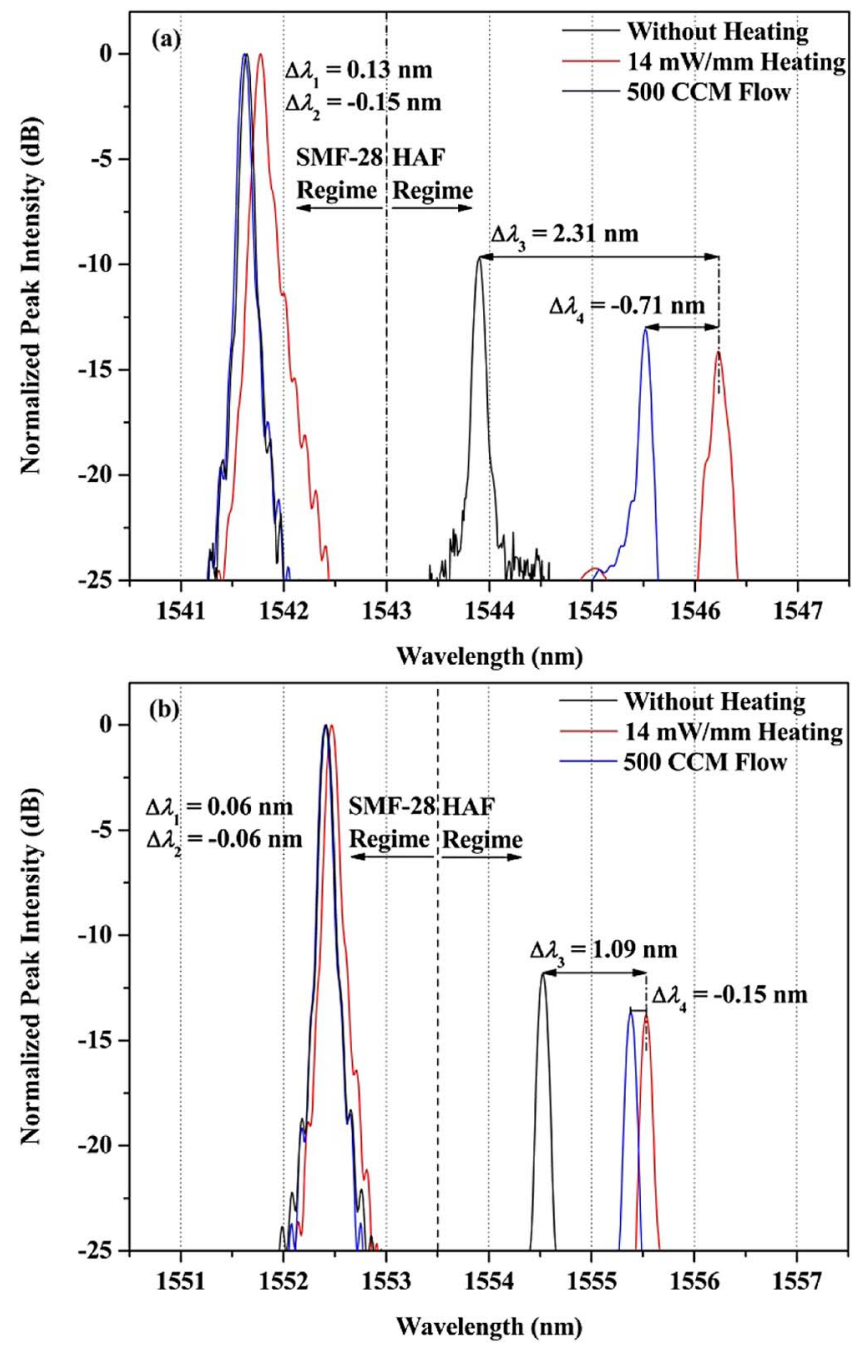

Fig. 5. Reflection spectra of both RFBGs in SMF-28 and HAF under different heating, flow conditions and ambient temperatures (a) $25^{\circ} \mathrm{C}$, (b) $800^{\circ} \mathrm{C}$.

RFBG in HAF and the reference temperature measured by the RFBG in SMF-28 can be combined to gauge the flow rate of $\mathrm{N}_{2}$.

For a HWA flow sensor, the temperature change from the heating element due to the gas flow can be related to the flow velocity $\nu$ by [4],

$$
H_{\text {power }}=\Delta T_{h}(A+B \sqrt{v}),
$$

where $H_{\text {power }}$ is the power absorbed by the heating element, $\Delta T_{h}$ is the temperature change from the ambient background on the HWA induced by an isothermal flow field, and $A$ and $B$ are empirical coefficients. In our case, $\Delta T_{h}$ is determined by the temperature changes on the heated RFBG in HAF and the ambient temperature measured by the reference RFBG in SMF-28 located at the upper stream of the gas flow according to,

$$
\Delta T_{h}=\left(\frac{d \lambda}{d T}\right)_{\mathrm{HAF}}^{-1}\left(\Delta \lambda_{3}+\Delta \lambda_{4}\right)+\left(\frac{d \lambda}{d T}\right)_{\mathrm{SMF}-28}^{-1}\left(\Delta \lambda_{1}+\Delta \lambda_{2}\right),
$$




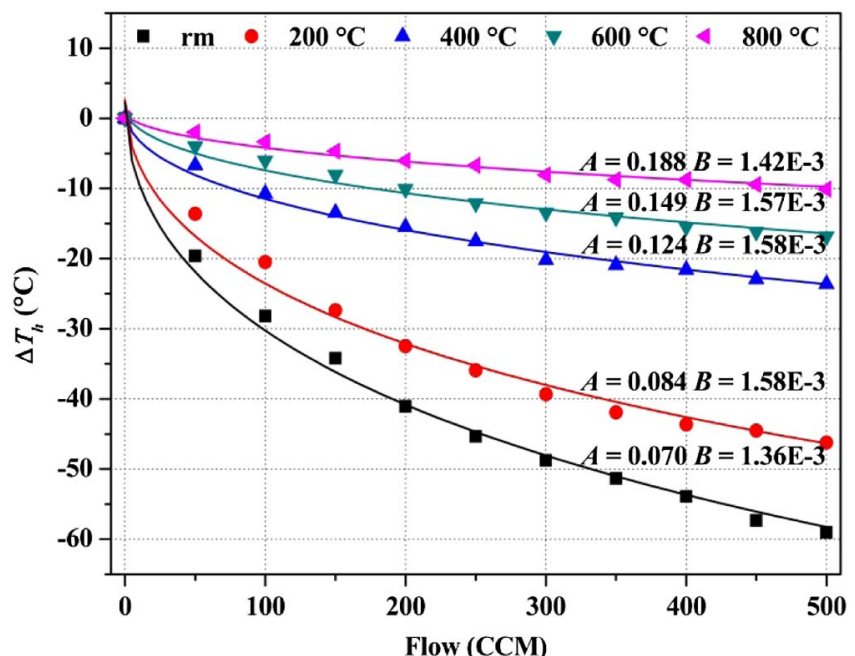

Fig. 6. Calibrated sensor responses of the flow under different ambient temperatures shown with fitted curves based on the HWA theory. A and B are coefficients of the fitting function with units of $\mathrm{mW} / \mathrm{mm} /{ }^{\circ} \mathrm{C}$ and $\mathrm{mW} / \mathrm{mm} /{ }^{\circ} \mathrm{C} /(\mathrm{m} / \mathrm{s})^{1 / 2}$ respectively.

where $(d \lambda / d T)_{\mathrm{HAF}}$ and $(d \lambda / d T)_{\mathrm{SMF}-28}$ are the wavelengthtemperature coefficients of RFBGs in both HAF and SMF-28 fibers, which are shown in Fig. 3. The accurate measurement of the ambient temperature by the reference RFBG is critical to ensure accurate flow measurements. This is because the introduction of gas flow can alter the ambient temperature in the vicinity of the HWA sensors [18].

The performance of the flow sensor under the maximum optical heating power $(14 \mathrm{~mW} / \mathrm{mm})$ was quantified and shown in Fig. 6 with fitted curves derived from (1). The empirical coefficients $A$ and $B$ of every fitted curve are also included in the figure. The fitted curves match well with the experimental flow measurements at all temperatures. This indicates effective operation of the allfiber hot-wire flow sensor operating under both the room temperature and at elevated temperatures. Due to the nature of HWA, the sensitivity of the sensor reduces at high temperatures due to lower heating efficiencies of the HAF. However, this problem can be remedied by increased optical heating power or using HAF with higher attenuation coefficients.

In conclusion, this Letter presents an all-optical hightemperature flow sensor based on HWA. Using in-fiber light as both the heating power and the interrogation light source, a pair of high-temperature-stable RFBGs were used to gauge the heat transfer from an optically powered heating element (HAF) induced by the gas flow. The entire flow sensor was constructed in one silica fiber and interrogated/powered through one fiber feed through. Reliable gas flow measurements were demonstrated for a wide temperature range from room temperature to $800^{\circ} \mathrm{C}$. The fiber sensor technique presented in this Letter provides a low-cost and reliable solution for flow sensing for high-temperature harsh environments.

This work was financially supported at the University of Pittsburgh by the Department of Energy National Energy Technology Laboratory through a grant (DE-FE0003859) and through a support contract with URS Energy \& Construction, Inc. under contract No. DE-FE00400.

\section{References}

1. Y.-H. Wang, C.-P. Chen, C.-M. Chang, C.-P. Lin, C.-H. Lin, L.-M. Fu, and C.-Y. Lee, Microfluid. Nanofluid. 6, 333 (2009).

2. G. D. Byrne, S. W. James, and R. P. Tatam, Meas. Sci. Technol. 12, 909 (2001).

3. O. Frazão, P. Caldas, F. M. Araújo, L. A. Ferreira, and J. L. Santos, Opt. Lett. 32, 1974 (2007).

4. H. H. Bruun, Hot-Wire Anemometry: Principles and Signal Analysis (Oxford University, 1995).

5. L. J. Cashdollar and K. P. Chen, IEEE Sens. J. 5, 1327 (2005).

6. C. Jewart, B. McMillen, S. K. Cho, and K. P. Chen, Sens. Actuat. A: Phys. 127, 63 (2006).

7. D. W. Lamb and A. Hooper, Opt. Lett. 31, 1035 (2006).

8. P. Caldas, P. A. S. Jorge, G. Rego, O. Frazão, J. L. Santos, L. A. Ferreira, and F. Araújo, Appl. Opt. 50, 2738 (2011).

9. W. Xinhuai, D. Xinyong, Z. Yan, N. Kai, C. Jia, and C. Zhemin, IEEE Photon. Technol. Lett. 25, 2458 (2013).

10. S. Gao, A. P. Zhang, H.-Y. Tam, L. H. Cho, and C. Lu, Opt. Express 19, 10124 (2011).

11. T. Chen, Q. Wang, B. Zhang, R. Chen, and K. P. Chen, Opt. Express 20, 8240 (2012).

12. Z. Bowei and K. Mojtaba, IEEE Sens. J. 7, 586 (2007).

13. S. Bandyopadhyay, J. Canning, M. Stevenson, and K. Cook, Opt. Lett. 33, 1917 (2008).

14. S. Bandyopadhyay, J. Canning, P. Biswas, M. Stevenson, and K. Dasgupta, Opt. Express 19, 1198 (2011).

15. K. Avila, D. Moxey, A. de Lozar, M. Avila, D. Barkley, and B. Hof, Science 333, 192 (2011).

16. E. Lindner, J. Canning, C. Chojetzki, S. Brückner, M. Becker, M. Rothhardt, and H. Bartelt, Appl. Opt. 50, 2519 (2011).

17. T. Wang, L.-Y. Shao, J. Canning, and K. Cook, Opt. Lett. 38, 247 (2013).

18. S. Takagi, J. Phys. E 19, 739 (1986). 